\title{
Research on the Design of a Gas Sampling Instrument
}

\author{
Xuezheng Zhu, Kunlin Nie, Xiaochun Hu, Huizhuo Li, Zhijie Wang \\ Institute of NBC Defence, Beijing, China, 102205
}

Keywords: gas sampler; sampling instrument; design

\begin{abstract}
Based on the requirements of an experiment, this paper designs a gas sampling instrument with distributed design method, and realizes the automatic collection of toxic samples and real-time communication in multiple points within an enclosed space, providing certain material conditions for the successful implementation of experiment.
\end{abstract}

\section{Introduction}

With the development and application of control technology, the intellectualization of gas sampling is improving continuously, and the design of special sampling instrument is a vital part of the successful implementation of special experiment. It is required to collect synchronously 250ml gas samples in multiple positions (P) and multiple time points (T) in an enclosed car body with no oxygen, that is PT samples with each one in $250 \mathrm{ml}$ ), for the analysis of gas composition by infrared detector and gas chromatograph. After the market exploration, it can be found that there is not ready-made sampling instrument suitable for this conditions, and it is necessary to customize it by design.

\section{Overview of Design Scheme}

The gas sampling instrument adopts distributed design, including collection unit and control unit, realizing communication by 485 trunk.

The collection unit is composed of mechanical structure, such as sample gasholder, sampling cylinder, electromagnetic mechanical device, gas pipeline, electromagnetic valve, etc., and control system, such as device power supply, control unit, intermediate relay, data communication unit, etc. External mechanical interface includes sampling air inlet and air outlet, and external electronic interface consists of power input port, two electrical interfaces for communication signals and operating light.

The control unit includes control circuit module, and LCD touch user interactive interface. External interface consists of power supply port, two electrical interfaces for communication signals, external trigger signal interfaces and operating light.

The control unit and collection unit adopt AC220V power supply, with two electrical interfaces for communication signals for each unit, which can realize cascading. After users set experiment parameters in LCD screen of control unit, the collection process can be started by LCD screen or external trigger signals.

\section{Technical Design Scheme}

In the design of this sampling instrument, technical contents involved mainly include actuator, electrical system and control system.

\subsection{Actuator}

The main body structure of collection unit pipeline in each sampling point is shown in Figure 1, taking $\mathrm{P}=6$ and $\mathrm{T}=6$ for illustration. Before the gas sampling, electromagnet is in power off, cylinder piston exhausts the air in the air cylinder with the force of spring, and all electromagnetic valves are normally-closed valves in power-off state. In gas sampling, power is connected in 
electronic control system for gasholder to correspond to electromagnetic valve, other five electromagnetic valves remain power off. Then the electromagnetic is connected to electricity to drive cylinder piston to inhale original air in gasholder into cylinder. External air to be sampled is inhaled into gasholder through sampling air inlet and one-way valve (The whole gas sampling process in completed with 1s). Then disconnect the electricity of electromagnetic valve, and gasholder is sealed. After the sampling, electromagnet is powered off, the spring drives cylinder piston, and the air in cylinder is exhausted from cylinder through one-way valve for the sampling of next time.

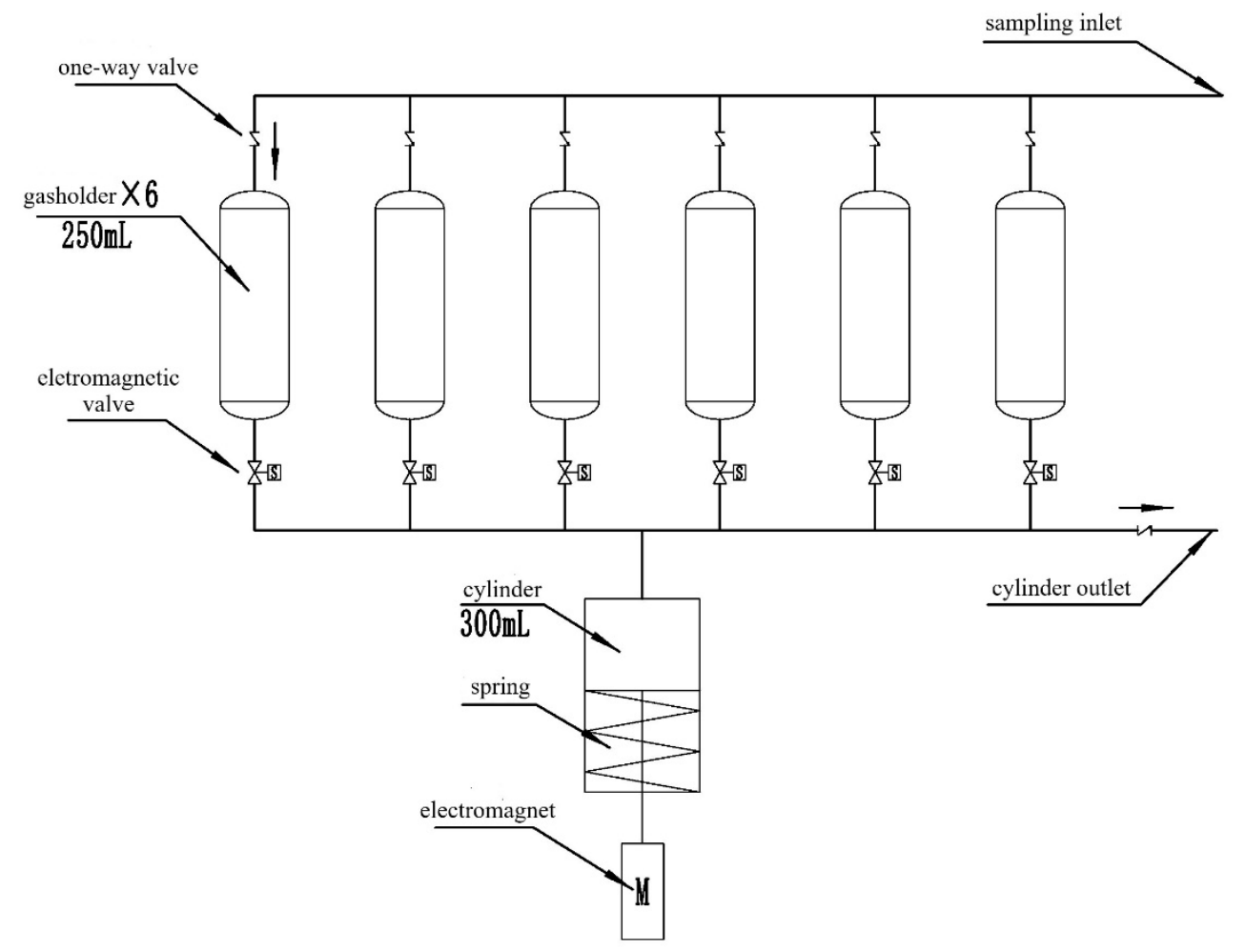

Figure 1 The main body structure of collection unit pipeline in each sampling point

The main body structure of gasholder is shown in Figure 2. There is detection port or extraction opening in gasholder, to put the probe of the sensor into gasholder to detect gas composition after the sampling, or connect sampler to collect samples for the analysis by gas chromatograph. The detection port adopts the structure of quick release and clamping, sealed by rubber ring; or sealed by screwed joint.

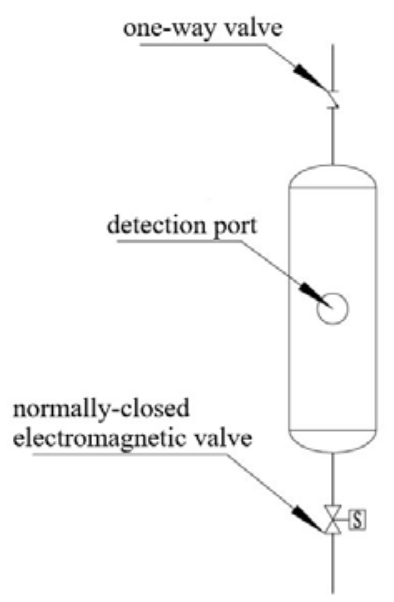


Figure 2 The main body structure of gasholder

\subsection{Electrical system}

The electrical system of collection unit is composed of control module, communication module, intermediate relay, switching power supply, and indication light, with modular design, DC12V battery as power supply, and 485 communication bus. The main body structure is shown in Figure 3.

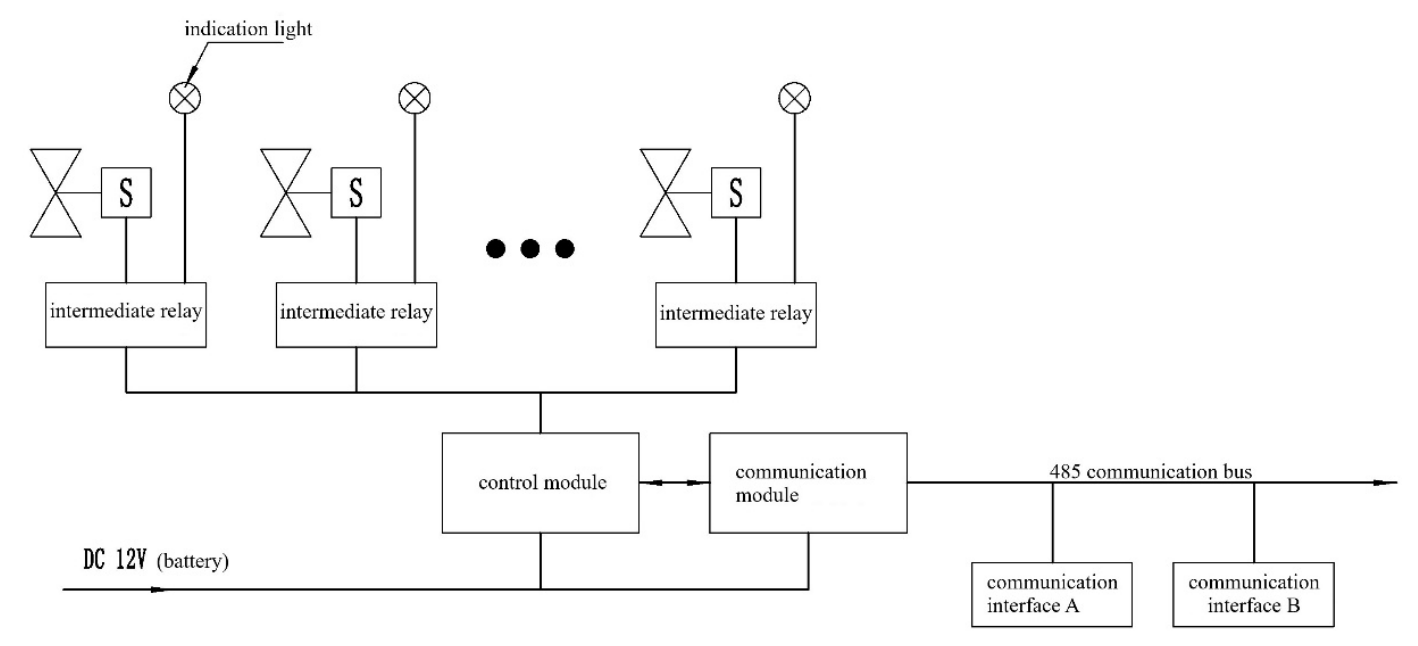

Figure 3 The main body structure of electrical system

\subsection{Control system}

The control module adopts DC12V battery as power supply, and communication module is connected to control unit through 485 bus. According to the instructions from control unit, control module drives intermediate relay, open corresponding electromagnetic valve or connect electricity to electromagnet and complete sampling or gas exhausting. Communication interface $\mathrm{A}$ and communication interface B are mounted to 485 bus for the signal connection of multiple modules. The control unit differentiates various collection units by the device address of built-in communication module in collection unit. The structure of control system is shown in Figure 4.

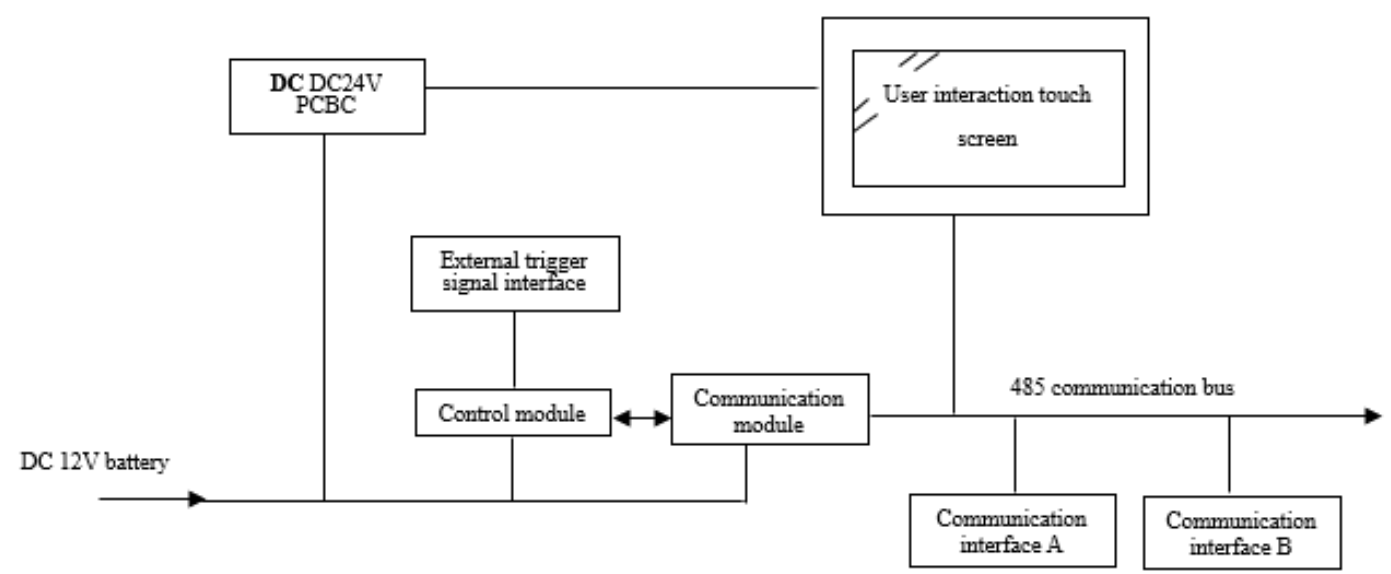

Figure 4 The structure of control system

The control system can realize user interaction interface through LCD touch screen, with built-in control module to collect external trigger signal to improve the expansibility of the instrument. Users can input parameters of gas sampling through touch screen, and then start sampling process by virtual button of touch screen or external trigger signal (or physical button or other switching signals). 


\section{Conclusion}

The system analysis and design in this paper, effectively solves the problems of gas sampling in multiple points and time in high risk environment, realizes the function of non-contact sampling in multiple points and time required by this experiment, meets user requirements, and is the successful application of computer technology and control technology in experimental technological field, which can be promoted in similar fields to effectively promote the greater development of experimental technology.

\section{References}

[1] Zhao Yaying, Yuan Yunping. Electrical Control System Design, Installation and Testing [M]. Beijing: Science Press, 2013.

[2] Wang Xiaohua, Lu Xinsheng. Pneumatic Components [M]. Beijing: China Machine Press, 1991.

[3] Xu Ke. Chemical Inspector (Senior) [M]. Beijing: Chemical Industry Press, 2009.

[4] Jinhai Yan, Weimin Han, Xibin Kao, et al.Test and Analysis on Harmful Gas in the Closed Cabin of a moving Special Vehicle[J]. Occupation and Health, 2014(6): 726-728

[5] Li Zhang, Jiang Liu, Honghua Zhang, et al. Discussion on the Test Method of harmful gases in cabins of tanks when hit by anti-tank weapons[J]. Med J Chin PLA, 2003(1):17-18 